\title{
Stage 0 Rectal Cancer AJCC v6 and v7
}

National Cancer Institute

\section{Source}

National Cancer Institute. Stage O Rectal Cancer A/CC v6 and v7. NCI Thesaurus. Code C4853.

Stage 0 includes: T is, N0, MO. T is: Carcinoma in situ: intraepithelial or invasion of the lamina propria. NO: No regional lymph node metastasis. MO: No distant metastasis. (AJCC 6th and 7th eds.) 